\title{
AF1q Expression Associates with CD44 and STAT3 and Impairs Overall Survival in Adenoid Cystic Carcinoma of the Head and Neck
}

\author{
Lorenz C. Kadletz ${ }^{1}$ (D) . Faris F. Brkic ${ }^{1} \cdot$ Bernhard J. Jank ${ }^{1} \cdot$ Sven Schneider ${ }^{1} \cdot$ Julia Cede $^{2} \cdot$ Rudolf Seemann $^{2}$. \\ Elisabeth S. Gruber ${ }^{3}$ • Elisabeth Gurnhofer ${ }^{4}$. Gregor Heiduschka ${ }^{1} \cdot$ Lukas Kenner $^{4,5,6}$
}

Received: 10 May 2019 / Accepted: 23 June 2019 / Published online: 4 July 2019

(C) The Author(s) 2019

\begin{abstract}
Salivary gland malignancies of the head and neck form a heterogeneous group. Adenoid cystic carcinomas are an aggressive entity of salivary gland malignancies characterized by frequent distant metastases and poor response to radio- and chemotherapy. AF1Q is a MLL fusion partner, which can activate Wnt and STAT3 signaling. Recently, overexpression of AF1q has been identified as a poor prognosticator in patients of different malignancies. A total of 46 patients with adenoid cystic carcinoma were immunohistochemically evaluated for expression of AF1q and clinical outcome was analyzed in this context. Additionally, STAT3 and the Wnt downstream target CD44 were investigated and correlated with AF1q. AF1q was overexpressed in $52.2 \%$. Overexpression of AF1q was associated with poorer overall survival $(p=0.03)$. Additionally, lymph node metastases and solid tumor parts were more frequently observed in AF1qhigh patients $(p=0.07$ and 0.05 , respectively). AF1q did not influence the occurrence of distant metastases. Expression of AF1q was associated with higher levels of STAT3 and CD44 ( $p=$ 0.003 and 0.006 , respectively). AF1q is a novel prognostic marker for poor overall survival in adenoid cystic carcinoma patients. The deleterious effects on survival may be a result of promotion of the STAT3 and Wnt pathway.
\end{abstract}

Keywords Adenoid cystic carcinoma $\cdot \mathrm{AF} 1 \mathrm{q} \cdot \mathrm{Wnt} \cdot \mathrm{STAT3} \cdot$ Prognosis

\section{Introduction}

Malignancies originating from salivary gland tissue are rare and have a reported incidence rate of 5-10 per 1,000,000. [1]

Gregor Heiduschka and Lukas Kenner contributed equally to this work.

Lorenz C. Kadletz

lorenz.kadletz@meduniwien.ac.at

Lukas Kenner

lukas.kenner@meduniwien.ac.at

1 Department of Otorhinolaryngology and Head and Neck Surgery, Medical University of Vienna, Vienna, Austria

2 Department of Craniomaxillo and Facial Surgery, Medical University of Vienna, Vienna, Austria

3 Department of General Surgery, Division of Surgery, Comprehensive Cancer Center, Medical University of Vienna, Vienna, Austria

4 Department of Experimental Pathology and Laboratory Animal Pathology, Medical University of Vienna, Vienna, Austria

5 Ludwig Boltzmann Institute for Cancer Research, Vienna, Austria

6 Department of Experimental Pathology and Laboratory Animal Pathology, University of Veterinary Medicine, Vienna, Austria
In addition, salivary gland malignancies appear as a heterogeneous group with several different histologic subtypes. Currently, the WHO differentiates between 22 malignant epithelial tumors of the salivary glands. [2] Both their scarcity and heterogeneity make it particularly difficult to study and understand all histologic subtypes.

Adenoid cystic carcinoma (ACC) confronts patients and clinicians with several serious issues and aggressive features that were first described by Dockerty and Mayo in 1942. [3] This kind of salivary gland carcinoma is characterized by distant metastases, high rate of recurrences and perineural invasion. Overall survival rates are reported to approximate $80 \%$ after 5 years and $60 \%$ after 10 years. [4] Nevertheless, over $40 \%$ of all ACC patients are confronted with distant metastases during the course of their disease. [5] Particularly patients diagnosed with the solid form of ACC are at a high risk of increased mortality and of developing distant metastasis. [6]

Park et al. have recently identified AF1q as a factor that promotes distant metastasis in breast cancer. [7] AF1q, a MLL fusion partner, was first described by Tse and colleagues as a fusion gene $(1 ; 11)(\mathrm{q} 21 ; \mathrm{q} 23)$ that is specifically expressed in leukemic and hematologic precursor cells. [8] Although the chromosomal abnormality that was detected then is not 
observed in solid tumors, AF1q expression has been found in various other malignancies including solid tumors. $[9,10]$ The oncogenic potential of AF1q may be a result of its capability to activate the Wnt pathway. [7] AF1q and its cofactors TCF1, beta-catenin and LEF1 form a transcriptional complex and activate the expression of CD44, a downstream target of the Wnt pathway. Aberrant Wnt signaling is associated with invasive characteristics of ACC in-vitro. [11] Activation of the Wnt pathway through beta catenin is associated with decreased survival rates in patients with major and minor salivary gland malignancies. [12, 13] Additionally, expression of AF1q enhances the oncogenic STAT3 signaling pathway via upregulation of PDGF-B and consecutive phosphorylation of the PDGF receptor beta. [14] Inhibition of STAT3 may be therefore a promising strategy in the treatment of ACC. Specifically, Bu and colleagues have demonstrated that selective inhibition of STAT3 by the small molecule inhibitor S3I201 results in decreased cellular proliferation, migration and invasion of ACC cell lines. [15]

In this context, we hypothesized that AF1q might be expressed in ACC of the head and neck. Moreover, we aimed to investigate its effects on patient survival and potential associations with the Wnt and STAT3 signaling pathways.

\section{Materials and Methods}

\section{Patients}

In this study patients with ACC that were operated on for ACC of major and minor salivary glands of the head and neck region at the Medical University of Vienna between 1996 and 2016 were included. Informed consent is obtained from every patient treated at the Vienna General Hospital that data may be used for studies. Inclusion criteria were met if the patient was diagnosed with ACC for the first time and aged over 18 years. Tracheal ACC were excluded from this study. TNM staging were assessed. Histologic reports were evaluated for grading according to Perzin/Szanto and Spiro depending on the predominant growth type (tubular, cribriform or solid) and for the presence of perineural and lymphovascular invasion. [16-18] Medical records including, especially time of initial diagnosis, last follow-up, death and local or distant recurrence were noted for survival time analysis. In addition, we assessed therapy at the time of initial diagnosis.

\section{Ethical Considerations}

This study was approved by the institutional research board of the Medical University of Vienna (ECS 1517/2018). All methods were performed in accordance with the Good Clinical Practice guidelines and the Declaration of Helsinki.

\section{Tissue Micro-Array and Immunohistochemistry}

Samples were collected from previously evaluated paraffinembedded ACC specimens that were obtained through surgical resection or biopsies taken prior to initial therapy via a Galileo Tissue Micro-Array (TMA) CK Series-HTS Tissue computer assisted Microarray Platform (Integrated Systems Engineering Srl, Milan, Italy).

Consecutively, $4 \mu \mathrm{m}$ sections were prepared and processed for immunohistochemical analysis.

Immunohistochemical stainings as well as histopathological analysis was performed by L.K. Immunohistochemical scores were calculated by adding up intensity $(0,1,2$ or 3$)$ and percentage of positive cells $(0-5 \%=0,5-40 \%=1,40$ $80 \%=2$ or $>80 \%=3$ ).

\section{Statistical Analysis}

Rates of overall survival (OS) and disease-free survival (DFS) were calculated by means of the Kaplan-Meier method. Statistical differences between groups were compared using the log-rank test (Mantel-Cox). Hazard ratio, standard error of the mean and confidence intervals were calculated. Univariate and multivariate analysis using a Cox regression model were performed additionally.

Categorical variables were compared using Fisher's exact test or $\mathrm{Chi}^{2}$ test. Mann-Whitney test was used for comparison of unpaired groups in case of ordinal data. In addition, ROC curves were created to calculate the Spearman correlation value r. A $p$ value $<0.05$ was considered as statistically significant.

SPSS software (Version 21.0; SPSS, Inc., Chicago, IL, USA) and Prism GraphPad software (GraphPad Software, Inc., La Jolla, CA, USA) were used to analyze and graphically present the data.

\section{Results}

\section{Patient Characteristics}

A total of 86 patients were diagnosed with ACC of the head and neck and treated at the Medical University of Vienna in the period between 1996 and 2016. Out of these, 46 patients had adequate medical records and quantities of material for immunohistochemical analysis. A total of 13 patients had small primary tumors (T1 $n=6$ and T2 $n=7$ ). (Table 1) Most of our patients had locally advanced disease. In 8 patients the tumor was classified as T3, and in the majority of all patients $(n=25)$ a T4 tumor was present at initial diagnosis. Lymph node metastases were detectable in 10 patients and distant metastases in 3 patients at the time of initial diagnosis. High-grade tumors according to Perzin and Szanto were 
Table 1 Basic data and descriptive statistics of patients with adenoid cystic carcinoma included in this study

\begin{tabular}{|c|c|c|c|c|c|}
\hline \multicolumn{3}{|c|}{ cystic carcinoma included $\mathrm{i}$} & & \multirow{2}{*}{ Number of patients } & \multirow{2}{*}{ Percentage } \\
\hline & & & & & \\
\hline & & & \multirow{2}{*}{$\begin{array}{l}\text { Solid\&Cribriform } \\
\text { Tubular\&Cribriform }\end{array}$} & \multirow{2}{*}{$\begin{array}{l}6 \\
9\end{array}$} & \multirow{2}{*}{$\begin{array}{l}13.0 \% \\
19.6 \%\end{array}$} \\
\hline Sex & & & & & \\
\hline Male & 20 & $43.5 \%$ & All three types & 2 & $4.3 \%$ \\
\hline Female & 26 & $56.5 \%$ & \multicolumn{3}{|l|}{ Localization } \\
\hline T-classification & & & Parotid Gland & 6 & $13.0 \%$ \\
\hline $\mathrm{T} 1$ & 6 & $13.0 \%$ & Submandibular Gland & 8 & $17.4 \%$ \\
\hline $\mathrm{T} 2$ & 7 & $15.2 \%$ & Sublingual Gland & 2 & $4.3 \%$ \\
\hline $\mathrm{T} 3$ & 8 & $17.4 \%$ & Minor Salivary Gland & 30 & $65.2 \%$ \\
\hline $\mathrm{T} 4$ & 25 & $54.4 \%$ & & & \\
\hline
\end{tabular}

N-classification

$\mathrm{N}+$

N0

10

36

M-classification

M0

M1

43

3

Perineural Invasion

Yes

No

23

23

Lymphovascular Invasion

Yes

No

Survival

Dead

Alive

Recurrence

Yes

No

Multiple recurrences

Yes

No

Treatment

Primary radiotherapy

Initial chemotherapy

Surgery 43

Postoperative radiochemotherapy

Postoperative radiotherapy

Perzin and Szanto Grading

1

2

3

Spiro Grading

1

2

3

Histologic components

Solid

Tubular

Cribriform

Solid\&Tubular
$21.7 \%$

$78.3 \%$

$93.5 \%$

$6.5 \%$

Table 1 (continued)

diagnosed in 9 patients. Five patients had more than $50 \%$ solid components and were therefore classified as high-grade according to the Spiro grading system. A total of 23 patients showed signs of perineural invasion, and lymphovascular invasion was microscopically detectable in 6 patients. During follow-up 25 patients died and 23 patients suffered from recurrent disease. In 18 patients distant metastases were found during their course of disease. Furthermore, 7 ACC patients were diagnosed with multiple recurrences during their course of disease. The vast majority of all patients $(n=43)$ was initially treated with surgical resection. Adjuvant therapy was applied in 20 patients.

$54.3 \%$

$45.7 \%$

$50.0 \%$

$50.0 \%$

$15.2 \%$

$84.8 \%$

$4.3 \%$

$2.2 \%$

$93.5 \%$

$23.3 \%$

$23.3 \%$

$26.1 \%$

$54.3 \%$

$16.6 \%$

$63.1 \%$

$23.9 \%$

$10.9 \%$

$8.7 \%$

$6.5 \%$

$46.7 \%$

$2.2 \%$

\section{Expression of AF1q and its Impact on Survival}

Out of 46 samples, AF1q expression was detected in 30 specimens, while 16 samples showed no signs of AF1q expression (Fig. 1a). Among the AF1q positive tumors, scores were distributed as follows: 6 patients had score 1-2 (low), 16 patients score 3-4 (moderate), and 8 patients score 5-6 (high) (Fig. 1b, $\mathrm{c}$ and $\mathrm{d})$. To gain more power for survival analysis patients were stratified into two groups, AF1q low- $($ score $=0-2)$ and high-expressing (score $=3-6$ ) tumors. Thus, a total of 22 patients were classified as $\mathrm{AF} 1 \mathrm{q}^{\text {low }}$ and 24 patients as $\mathrm{AF} 1 \mathrm{q}^{\text {high }}$.

First of all, we investigated the effects of AF1q in the context of several clinicopathologic parameters. As shown in Table 2, T1-4 tumors were equally distributed between both groups. Lymph node metastases were more often detected in patients with high expression of AF1q in primary tumors (9.1\% vs. AF1 $\left.q^{\text {high }} 33.3 \%\right)$. However, results were not statistically significant $(p=0.07)$. As AF1q promotes the formation of distant metastases in breast cancer patients [7], we compared the incidence of distant metastases at initial diagnosis and distant recurrences in dependence of AF1q expression. Surprisingly, we did not find any differences in the occurrence of distant metastases at different time points $(p>0.99$ and $p=0.77$ for initial diagnosis and distant recurrence, respectively). Solid components of ACC are usually associated with poor prognosis. In our study, solid parts were $>3$ times more often observed in AF1 $\mathrm{q}^{\text {high }}$ tumors $(41.7 \%$ vs. $13.6 \%$ ). 
Fig. 1 Samples of patients with adenoid cystic carcinoma (ACC) and predominately tubular growth pattern are shown in (a) and (b). In (a) no expression of AF1q could be observed and in (b) very low intensity could be observed in $<5 \%$ of all tumor cells. Sample (c) shows a mixed growth pattern with moderate expression of AF1q. High expression of a solid typ is depicted in sample (d)

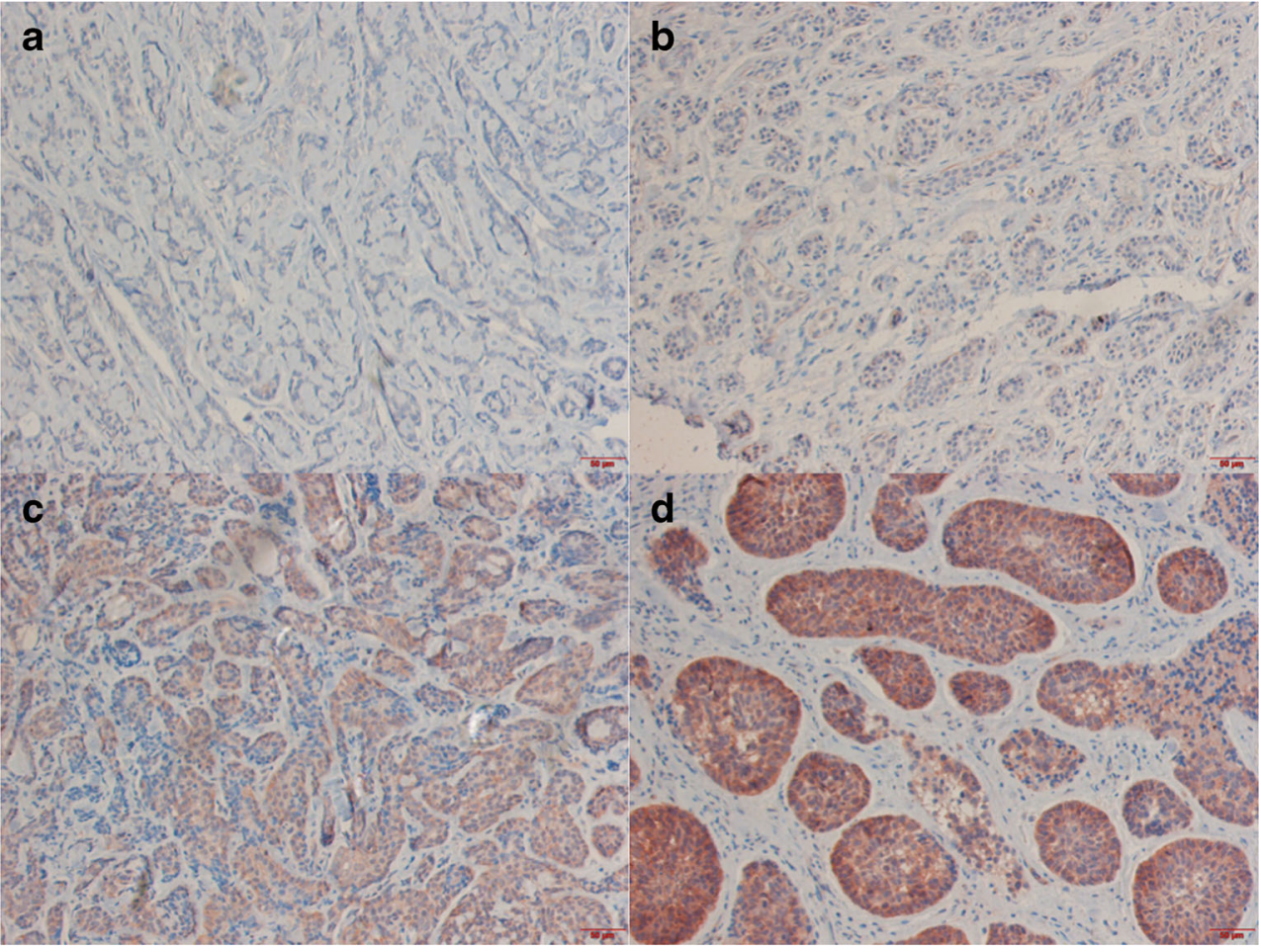

Nevertheless, the level of significance was not reached $(p=$ 0.051 ). Although solid components were more frequently detected, high-grade tumors were not associated with AF1q expression in dependence of the Perzin/Szanto grading system $(p=0.70)$. Interestingly, patients with more than $50 \%$ of solid tumor tissue were only detected in the AF1 $\mathrm{q}^{\text {high }}$ group and

Table 2 Comparison and statistical analysis of AF1 $\mathrm{q}^{\text {low }}$ and $\mathrm{AF} 1 \mathrm{q}^{\text {high }}$ patients

\begin{tabular}{lrcc}
\hline & AF1q $^{\text {low }}$ & AF1 q & p value \\
\hline Total number of patients & 22 & 24 & \\
T-classification & & & 0.9368 \\
T1 & 3 & 3 & \\
T2 & 4 & 3 & \\
T3 & 4 & 4 & \\
T4 & 11 & 14 & \\
Lymph node metastases & 2 & 8 & 0.0740 \\
Distant metastases (initially) & 1 & 2 & 0.9999 \\
Distant recurrences & 8 & 10 & 0.7693 \\
Perineural Invasion & 11 & 12 & 0.9999 \\
Solid components & 3 & 10 & 0.0509 \\
High Grad Malignancy & & & \\
Perzin/Szanto & 3 & 5 & 0.7021 \\
Spiro & 0 & 5 & 0.0502 \\
Localized in Minor Salivary Glands & 15 & 15 & 0.7628 \\
Death & 6 & 19 & 0.0009 \\
Recurrence & 10 & 13 & 0.7683 \\
\hline
\end{tabular}

strictly solid tumors were just found within this group as well. However, statistical significance was missed $(p=0.0502)$. Perineural invasion was equally distributed in the $\mathrm{AF} 1 \mathrm{q}^{\text {low }}$ and AF1 $\mathrm{q}^{\text {high }}$ tumor group $(p>0.99)$.

Survival analysis revealed a mortality rate almost three times higher in patients with AF1 qigh tumors (79.1\% vs. $27.3 \%, p=0.001$ ), whereas the recurrence rate was not significant in patients with $\mathrm{AF} 1 \mathrm{q}^{\text {high }}$ tumors $(54.2 \%$ vs. $45.4 \%, p=$ 0.77). Kaplan-Meier curves showed a median OS in patients with $\mathrm{AF} 1 \mathrm{q}^{\text {low }}$ tumors (140.3 months) and in patients with AF1 $q^{\text {high }}$ tumors (75.7 months). Log-rank tests showed that high expression of AF1q significantly worsened OS in ACC patients $(p=0.03)$. Multivariate analysis revealed a significant difference on distant metastases and T1 vs. T4 tumors (data not shown). Analysis of DFS indicated no significant difference between both groups (AF1 $\mathrm{q}^{\text {high }} 51.6$ months vs. AF1 $\mathrm{q}^{\text {low }}$ 51.1 months, $p=0.77$ ). (Fig. $2 \mathrm{a}, \mathrm{b}$ ).

\section{AF1q Expression Is Associated with Up-Regulation of CD44 and STAT3}

Previous studies showed an association of AF1q expression with expression of STAT3 and the Wnt downstream target CD44.

The group of patients with AF1 $\mathrm{q}^{\text {high }}$ tumors included 9 patients (34.6\%) with a STAT3 score of 6 and 12 patients (46.2\%) with a STAT3 score of 5. (Fig. 3). In contrast, only 2 patients $(9.1 \%)$ in the AF1q ${ }^{\text {low }}$ group had a STAT3 score of 6 and 9 patients (40.9\%) a score of 5. Mann-Whitney tests revealed that STAT3 was significantly higher expressed in 


\section{a Overall Survival}

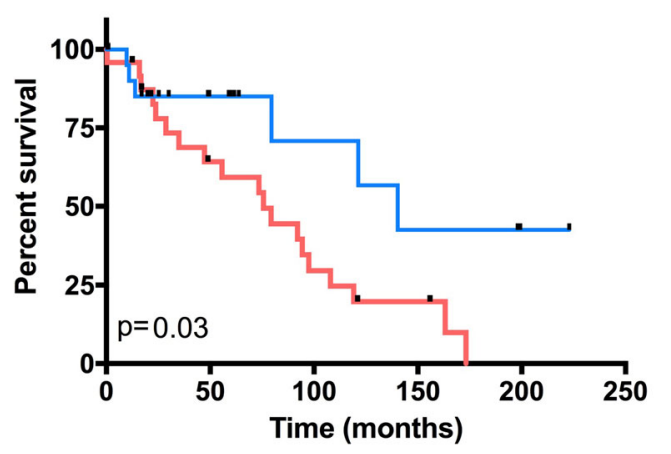

b Disease-free Survival

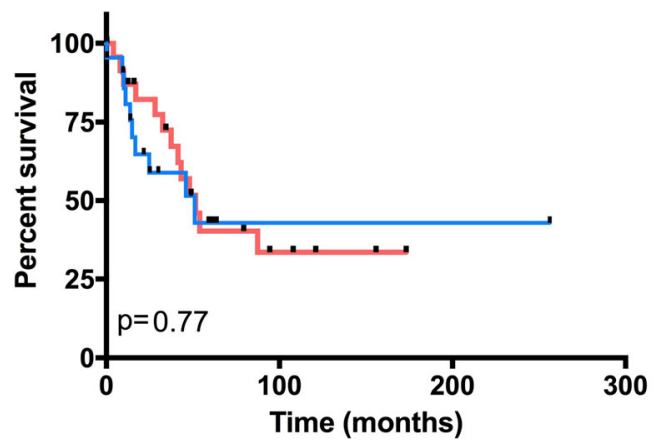

Fig. 2 Patients were stratified into AF1 $\mathrm{q}^{\text {low }}$ and $\mathrm{AF} 1 \mathrm{q}^{\text {high }}$. Overall survival in dependence of AF1q expression is shown in (a). Median survival in AF1 $\mathrm{q}^{\text {low }}$ measured 140.3 months and 75.7 months in AF1q ${ }^{\text {high }}(p=0.03)$. Disease free survival (b) showed no significant difference in outcome $(p=0.77)$

$$
\begin{aligned}
& +A F 1 q^{\text {low }} \\
& +A F 1 q^{\text {high }}
\end{aligned}
$$

AF1 $q^{\text {high }}$ patients $(p=0.002)$. Next, ROC curves were computed, revealing a moderate correlation of STAT3 with AF1q expression (Spearman $r$ correlation coefficient $=0.53$ ).

CD44 showed lower expression levels than STAT3 in our cohort. Only 2 patients $(7.7 \%)$ expressed CD44 with a score of 6 in the AF1q ${ }^{\text {high }}$ group, and no patient with a score of 6 was detected in the AF1 $q^{\text {low }}$ group. No expression of CD44 was found in 6 patients (23.1\%) with AF1 $\mathrm{q}^{\text {high }}$ expression levels and in 13 patients $(59.1 \%)$ with $A F 1 q^{\text {low }}$ expression. Again, Mann-Whitney test showed that AF1 ${ }^{\text {high }}$ patients had a significantly higher expression of $\mathrm{CD} 44$, with a moderate correlation being observed between AF1q and CD44 expression (Spearman $r$ correlation coefficient $=0.44$ ).

\section{Discussion}

In our study expression of AF1q was immunohistochemically detectable in almost two thirds of the patients, and more than half of our cohort expressed AF1q at a high or moderate level. Expression of AF1q was found in a number of other solid malignancies such as breast cancer, ovarian cancer, and colorectal cancer. [7, 19,20] Subsequently, we compared survival of patients with AF1 $\mathrm{q}^{\text {high }}$ and AF1 $\mathrm{q}^{\text {low }}$ tumors in our study. One of the major findings was that patients with $\mathrm{AF} 1 \mathrm{q}^{\text {high }}$ tumors showed a significant decrease in OS. Other studies reported similar findings. Hu and colleagues were able to demonstrate via The Cancer Genome Atlas dataset of colorectal cancer patients that AF1q expression resulted in reduced
Fig. 3 Examples of AF1q (a), CD44 (b) and STAT3 (c) expression patterns observed in the same patient. Additionally, AF1q ${ }^{\text {high }}$ patients showed significant higher co-expression of CD44 (D) and STAT3 (e)

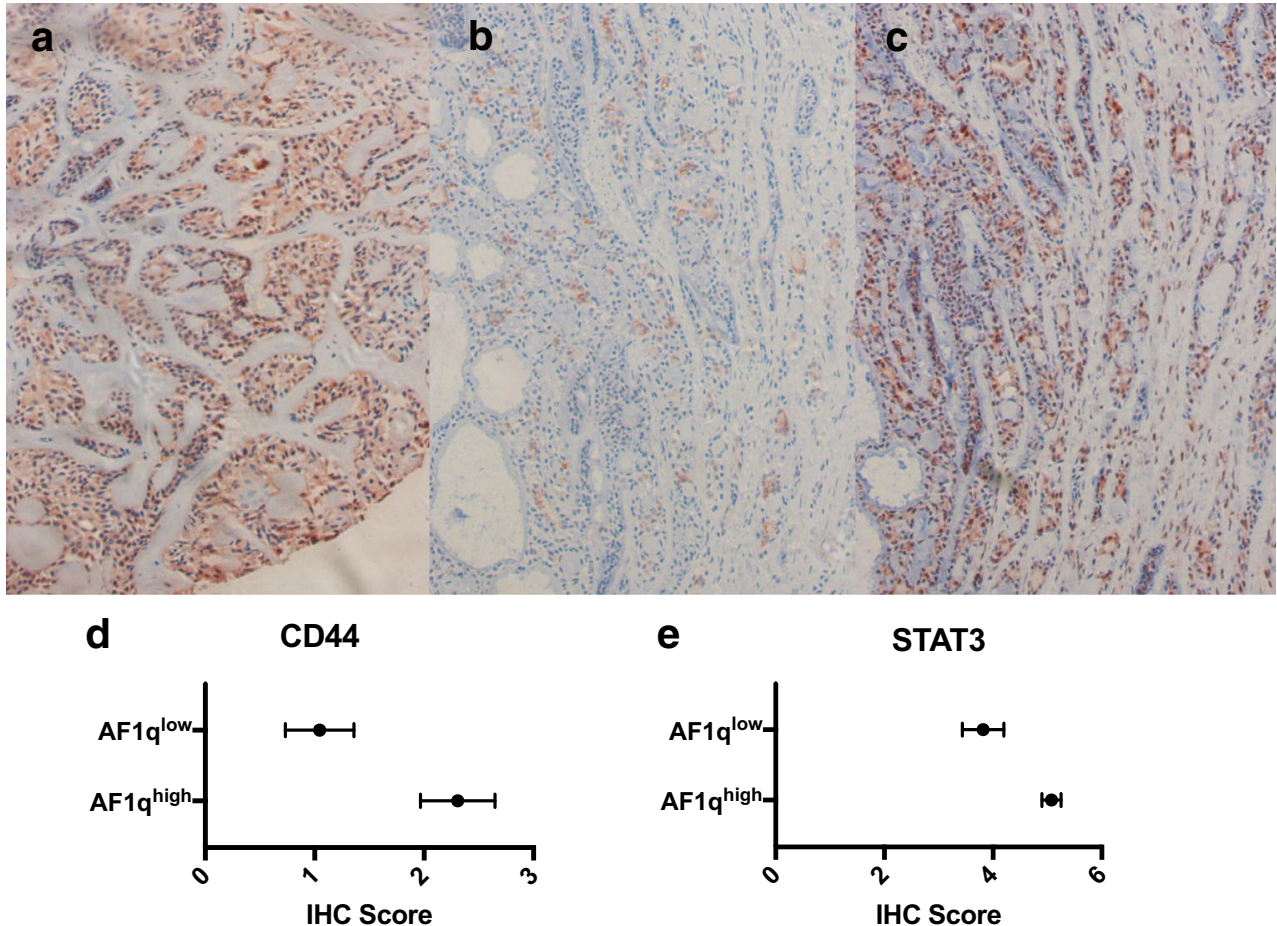


OS and DFS rates. Park and coworkers reported similar results in breast cancer patients. [7] In addition, their study group was able to identify AF1q as a prognosticator for brain metastases. Interestingly, we did not find any difference in the occurrence of distant metastases at the time of initial diagnosis or in the course of disease. Nevertheless, lymph node metastases were detected $>3$ times more often in patients with $\mathrm{AF} 1 \mathrm{q}^{\text {high }}$ tumors. However the level of significance was not reached. The fact of AF1q not being associated with metastatic disease might be contributed to the low number of patients eligible for this analysis. In a study investigating 96 colorectal cancer patients, AF1q upregulation was significantly associated with lymph node metastases [19] Moreover, expression of AF1q was predominately found in patients with solid components of ACC. A solid growth pattern in ACCs is generally associated with worse clinical outcome. Strictly solid ACCs were only found in $\mathrm{AF} 1 \mathrm{q}^{\text {high }}$ patients.

In addition, we examined the expression of $\mathrm{CD} 44$, as a downstream target of the Wnt pathway, and of STAT3 in our cohort. Our data suggests that AF1q expression may lead to an activation of both pathways since CD44 and STAT3 are expressed at a higher intensity in AF1 ${ }^{\text {high }}$ patients. The promoting role of AF1q for the oncogenic STAT3 pathway was described by Park et al. [14] Furthermore, AF1q has been shown to induce expression of PDGF-B/PDGFBR-B, which results in a markedly enhanced STAT3 activity. Additionally, AF1q results in the transcriptional activation of CD44 downstream of Wnt. [7] Activated Wnt signaling has recently been associated with AF1q activation and the enhancement of cancer stem cell population in breast cancer. [21]

In conclusion, we have been able to link the expression of AF1q in patients with ACC to a significant decrease in OS. Our results could not confirm higher rates of distant metastases in ACC patients compared to other data reported in the literature. However, lymph node metastases were more often found in patients $\mathrm{AF} 1 \mathrm{q}^{\text {high }}$ tumors. Additionaly, we linked AF1q expression to higher levels of STAT3 and CD44. As a matter of fact, association with the STAT3 as well as the Wnt signaling pathway might open new therapeutic perspectives in fighting ACC. Taken together, our results argue in favor of further investigation of AF1q in ACC of the salivary glands in order to confirm our results and to examine AF1q as a potential therapeutic target.

Funding Information Open access funding provided by Medical University of Vienna.

\section{Compliance with Ethical Standards}

Conflict of Interest All authors state to have no conflict of interest and declare to have neither financial nor non-financial competing interests.
Open Access This article is distributed under the terms of the Creative Commons Attribution 4.0 International License (http:// creativecommons.org/licenses/by/4.0/), which permits unrestricted use, distribution, and reproduction in any medium, provided you give appropriate credit to the original author(s) and the source, provide a link to the Creative Commons license, and indicate if changes were made.

\section{References}

1. Sood S, McGurk M, Vaz F (2016) J Larnygol Otol 130:S142

2. Barnes L, Eveson JW, Reichart P, Sidransky D (2005) Pathology and genetics of head and neck Tumours. IARC Press

3. Dockerty MB, Mayo CW (1942) Surg Gynecol Obstet 1033

4. Lloyd S, Yu JB, Wilson LD, Decker RH (2011) Am J Clin Oncol 34:76

5. van Weert S, Reinhard R, Bloemena E, Buter J, Witte BI, Vergeer MR, Leemans CR (2016) Head Neck 39:456

6. Fordice J, Kershaw C, El-Naggar A, Goepfert H (1999) Arch Otolaryngol Head Neck Surg 125:149

7. Park J, Schlederer M, Schreiber M, Ice R, Merkel O, Bilban M, Hofbauer S, Kim S, Addison J, Zou J, Ji C, Bunting ST, Wang Z, Shoham M, Huang G, Bago-Horvath Z, Gibson LF, Rojanasakul Y, Remick S, Ivanov A, Pugacheva E, Bunting KD, Moriggl R, Kenner L, Tse W (2015) Oncotarget 6(20697)

8. TSE W, ZHU WM, CHEN HS, COHEN A (1995) Blood 85:650

9. Co NN, Tsang WP, Tsang TY, Yeung CLA, Yau PL, Kong SK, Kwok TT (2010) Oncol Rep 24:547

10. Tiberio P, Cavadini E, Callari M, Daidone MG, Appierto V (2012) PLoS One 7:e39968

11. Wang R, Geng N, Zhou Y, Zhang D, Li L, Li J, Ji N, Zhou M, Chen Y, Chen Q (2015) Biomed Mater Eng 26:S2145

12. Schneider S, Thurnher D, Seemann R, Brunner M, Kadletz L, Ghanim B, Aumayr K, Heiduschka G, Lill C (2015) Eur Arch Otorhinolaryngol 273:1283

13. Lill C, Schneider S, Seemann R, Kadletz L, Aumayr K, Ghanim B, Thurnher D (2014) Head Neck 37:30

14. Park J, Kim S, Joh J, Remick SC, Miller DM, Yan J, Kanaan Z, Chao J-H, Krem MM, Basu SK, Hagiwara S, Kenner L, Moriggl R, Bunting KD, Tse W (2016) Oncotarget 7(43960)

15. Bu L-L, Deng W-W, Huang C-F, Liu B, Zhang W-F, Sun Z-J (2015) Am J Cancer Res 5:1751

16. Spiro RH, Huvos AG, Strong EW (1974) Am J Surg 128:512

17. Szanto PA, Luna MA, Tortoledo ME, White RA (1984) Cancer 54: 1062

18. Perzin KH, Gullane P, Clairmont AC (1978) Cancer 42:265

19. Hu J, Li G, Liu L, Wang Y, Li X, Gong J (2017) Int J Mol Sci 18

20. Tiberio P, Lozneanu L, Angeloni V, Cavadini E, Pinciroli P, Callari M, Carcangiu ML, Lorusso D, Raspagliesi F, Pala V, Daidone MG, Appierto V (2017) Oncotarget 8(23246)

21. Tse CO, Kim S, Park J (2017) Biochem Biophys Res Commun 484: 884

Publisher's Note Springer Nature remains neutral with regard to jurisdictional claims in published maps and institutional affiliations. 